\title{
Gastric Outlet Obstruction due to Cytomegalovirus Infection in an Immunocompromised Patient
}

\author{
Vasileios Panteris $^{\mathrm{a}} \quad$ Antigoni Karakosta $^{\mathrm{b}}$ \\ Emmanuel Merikas $^{a} \quad$ George Peros $^{c} \quad J o h n$ K. Triantafillidis ${ }^{a}$ \\ Departments of a Gastroenterology and 'bathology, 'Saint Panteleimon' Hospital, \\ Nicea, and '4th Surgical Unit, 'Attikon' Hospital, Haidari, University of Athens, \\ Athens, Greece
}

\section{Key Words}

Cytomegalovirus · Gastritis · Gastric ulcer · Ganciclovir · Endoscopy · Gastric outlet obstruction

\begin{abstract}
A case of gastric outlet obstruction accompanied by diffuse gastritis and gastric ulcer due to cytomegalovirus infection is presented. The patient, a woman aged 67 years with a long history of rheumatoid arthritis under immunosuppressive treatment (methotrexate), was admitted to our department complaining mostly of abdominal pain located in the epigastrium and the right abdomen. Upper gastrointestinal endoscopy revealed the presence of gastritis accompanied by ulcer in the prepyloric area and gastric outlet obstruction due to cytomegalovirus infection which was confirmed histologically. The patient responded well - although after many weeks - to specific treatment with antiviral treatment (ganciclovir). It is concluded that gastric outlet obstruction caused by cytomegalovirus infection can be observed in immunocompromised patients. The clinician must search for possible cytomegalovirus infection in all immunocompromised patients presenting with a clinical picture of gastric outlet obstruction by obtaining enough biopsies and by asking the histopathologist to specifically stain the specimen for the presence of cytomegalovirus, especially if infection by Helicobacter pylori is not present.
\end{abstract}

\section{Introduction}

Cytomegalovirus (CMV) infection is a common cause of morbidity and mortality among patients receiving immunosuppressive treatment. Gastrointestinal infection by 
CMV includes mainly involvement of the large bowel, especially in patients with underlying inflammatory bowel disease. Gastritis caused by CMV infection in immunocompromised patients, although quite rare, seems to be important from both a clinical and therapeutic point of view [1,2]. Moreover, diffuse gastritis due to CMV infection has been described in bone marrow transplant patients [3], in patients after cord blood transplantation for acute myeloid leukemia [4] and after rituximab treatment for non-Hodgkin's lymphoma [5]. It must be stressed however that development of CMV gastritis and gastric ulcer has also been noticed in immunocompetent subjects $[6,7]$.

Gastric outlet obstruction (GOO) due to CMV infection has very rarely been described [8]. We present a woman with long-standing rheumatoid arthritis who developed diffuse gastritis accompanied by gastric ulcer and GOO due to CMV infection while receiving methotrexate and non-steroidal anti-inflammatory drugs for a long period of time.

\section{Case Report}

A woman aged 67 years was admitted to our department because of abdominal pain located mainly in the epigastrium and right side of the abdomen accompanied by weight loss and fatigue. The symptoms had appeared eight months before with a clinical episode compatible with gastroenteritis. Personal history included rheumatoid arthritis and the patient had been on regular treatment with methotrexate for more than twelve years. During the last few months she had also been receiving non-steroidal anti-inflammatory drugs on demand. She was a current smoker. On physical examination, except for some tenderness on palpation of the epigastrium and right abdomen, no significant findings were noticed. Initial investigations revealed low hemoglobin concentration $(9.5 \mathrm{~g} / \mathrm{l})$ and hematocrit $(31 \%)$, increased erythrocyte sedimentation rate $(39 \mathrm{~mm} / 1 \mathrm{st} \mathrm{h})$ and fibrinogen $(411 \mathrm{mg} / \mathrm{dl})$, increased CRP and al-acid-glycoprotein (17 and $1.44 \mathrm{~g} / \mathrm{l}$, respectively) and low serum albumin $(3 \mathrm{~g} / \mathrm{l})$. Liver and thyroid function tests were normal. Serum tumor markers, gastrin, vitamin $B_{12}$ and folic acid were normal. Blood and stool cultures were negative. Microscopic examination of stool specimen was unremarkable. IgG and IgA antibodies against Yersinia enterocolitica [Yop M (2a)] (Western blot) were negative. Chest X-ray was normal. Serum IgG anti-CMV antibodies were positive while serum IgM anti-CMV antibodies were negative. Serum IgG and IgA levels were low. Antinuclear and pANCA antibodies were positive (table 1).

A computerized tomography scan (hydro-CT) of the abdomen revealed thickening of the gastric wall located mainly in the antrum. Transabdominal ultrasonography revealed normal findings. Upper gastrointestinal endoscopy demonstrated multiple linear exudative gastric ulcers, the largest being $3 \mathrm{~cm}$ in diameter. The pylorus was edematous and narrowed. The $9 \mathrm{~mm}$ endoscope was finally passed through the pylorus, although with great difficulty. Colonoscopy performed at that time revealed some aphthous ulcers in the terminal ileum.

Histology of the terminal ileum revealed only mild inflammatory infiltration and lymphoid hyperplasia. Histological examination of specimens obtained from the gastric ulcer, the surrounding area and other parts of the stomach revealed the presence of diffuse gastritis. Abundance of hyperchromatic epithelial cells with nuclear viral inclusions (owl's eye) was demonstrated. These cells were positive for monoclonal antibodies against CMV (fig. 1). Helicobacter pylori could not be demonstrated in the gastric biopsies. Repeat upper gastrointestinal endoscopy performed few days later because of worsening of symptoms, as well as histology of gastric specimens, confirmed the previous results. However, this time and despite great efforts, passing the endoscope through the pylorus was impossible.

Consequently, treatment with intravenous ganciclovir $(2 \times 5 \mathrm{mg} / \mathrm{kg} / \mathrm{day})$ was initiated with slow clinical improvement seen after 3-week treatment. However, after 6 weeks continuous treatment her symptoms resolved almost completely and on a subsequent endoscopy only signs of healing ulcers were seen. Colonoscopy performed again after 2 months was unremarkable. 


\begin{tabular}{r|l|l|l}
$\begin{array}{r}\text { Case Reports } h \\
\text { Gastutuenterclagy }\end{array}$ & $\begin{array}{l}\text { Case Rep Gastroenterol 2009;3:280-285 } \\
\text { D01: 10.1159/000228895 }\end{array}$ & Published online: September 12, 2009 & $\begin{array}{l}\text { @ 2009 S. Karger AG, Basel } \\
\text { ISSN 1662-0631 } \\
\text { www.karger.com/crg }\end{array}$ \\
\hline
\end{tabular}

\section{Discussion}

The intriguing element in this case is the atypical manifestation of the patient's disease state, namely GOO accompanied by severe gastric ulceration without the heralding symptoms of emesis, inability to eat, bloating, early satiety and epigastric fullness. The interest in this case is the clinical scenario in which CMV, although a common viral factor especially in immunocompromised patients, was not an asymptomatic bystander, but instead was etiologically associated with the disease. The present case obviously leads to the assumption that the appearance of GOO in immunocompromised patients must guide the physician not to proceed immediately with surgical restoration of the continuity of the gastrointestinal tract, but to wait for the results of the histology. After confirmation of diagnosis of CMV infection, the physician must also wait for the results of the specific antiviral treatment before considering a surgical approach.

Our case also raised some diagnostic dilemmas because of the absence of pathognomonic endoscopic features for CMV infection and also created difficulties concerning both the right medical treatment and the best way of follow-up. The message for the clinical gastroenterologist is to search for the possibility of the existence of CMV infection in immunocompromised patients with a clinical picture compatible with GOO by obtaining a large number of biopsies and by asking specifically for CMV staining, especially if infection by Helicobacter pylori is not present.

An important point regarding the diagnosis and course of CMV infection could be the number of infected cells in the biopsy specimens in relation to the severity of endoscopic lesions. In a relevant study, no correlation between CMV inclusion bodies count and severity of symptoms or length of survival could be demonstrated [9]. On the other hand, coexistence of other potential pathogens, such as herpes simplex, mycobacteria and candida, could - at least theoretically - further complicate the clinical course [9]. The coexistence of other infective agents was excluded by clinical and laboratory findings (normal chest X-ray, negative Mantoux test) as well as special staining of biopsy specimens (Ziel Nielsen-PAS).

Another interesting point in this case was the negative serum IgM anti-CMV antibodies despite the presence of CMV in the gastric biopsies. However, it is generally accepted that in patients with active CMV infection, negative serologic test can be obtained as this test is an unreliable marker of diagnosis of current infection due to low sensitivity [10].

CMV gastritis may appear in non-immunosuppressed individuals as well. Vergara et al. [6] described two immunocompetent patients with gastric ulcer as the only manifestation of CMV infection. Both patients required antiviral treatment, and one case evolved to GOO requiring surgery. The latter case, as well as the case described by Mohammed et al. [8] and our own, are probably the only cases of CMV gastritis accompanied by GOO described so far in the literature.

Some descriptions suggest that the clinical picture of CMV gastritis is dominated by epigastric pain having some special characteristics: it is decreasing in supine position of the patient and it is increasing while sitting, standing or walking [2]. In our patient the cardinal symptom was also abdominal pain mainly located in the epigastrium and having the same characteristics as those described in the literature.

Diagnosis of CMV gastritis can be very difficult if one relies only on the findings of upper gastrointestinal endoscopy as the endoscopic picture is not characteristic [11]. The protean radiologic features are not specific either and may mimic several other conditions 
[12]. It has been suggested that diagnosis of CMV gastritis can be facilitated by the use of qualitative PCR. However, we suggest that clinical suspicion plays the most important role in making the correct diagnosis. The clinician should always consider CMV infection as a possible cause of gastritis, especially in immunosuppressed patients. Consequently, the endoscopist must advise the histopathologist accordingly in order to search for the presence of CMV infection.

Data concerning the natural history of CMV gastritis are not available. The pathophysiological consequences of CMV gastritis are not well understood either, although reduced gastric emptying [13] and dysmotility [14] have been described. In all cases of CMV gastritis described so far, epigastric pain completely resolved after treatment with ganciclovir [1], as it happened in our patient. Prolonged low-dose ganciclovir prophylaxis after heart transplantation reduces the incidence of CMV disease in CMV-mismatched patients and reduces the morbidity of CMV disease in CMV-positive recipients [15].

We cannot answer the question whether the aphthous ulcers seen in the terminal ileum in the first colonoscopy were actually part of a disseminated CMV infection or the result of the consumption of NSAIDs. Histology did not confirm the presence of CMV infection. However, the disappearance of the lesions in the follow-up colonoscopy after completion of anti-CMV treatment might be a clue to CMV ileitis.

\section{Conclusion}

GOO caused by CMV infection can be noticed in immunocompromised patients. The clinician must search for the presence of CMV infection in all immunocompromised patients presenting with a clinical picture of GOO by obtaining enough biopsies and by asking the histopathologist to specifically stain the specimen for CMV infection, especially if infection by Helicobacter pylori is not present.

Table 1. Results of serum immunological parameters

\begin{tabular}{ll}
\hline Parameter & Initial value \\
\hline ANA & positive in low titer \\
RA test & negative \\
Serum immunoglobulins & low IgG and IgA \\
Immunoblotting & absence of paraprotein \\
pANCA & positive \\
IgG CMV (serum) & positive $(32)$ \\
& [positive $>11 \mathrm{AU} / \mathrm{ml}$ ] \\
IgM CMV (serum) & negative $(1.13)$ \\
& [positive $>11 \mathrm{AU} / \mathrm{ml}$ ] \\
\hline
\end{tabular}


Fig. 1. Gastric epithelium $\mathrm{CMV}(+), 60 \times$. Immunohistochemistry with monoclonal anti-CMV (CMV Ab1, clone 01).

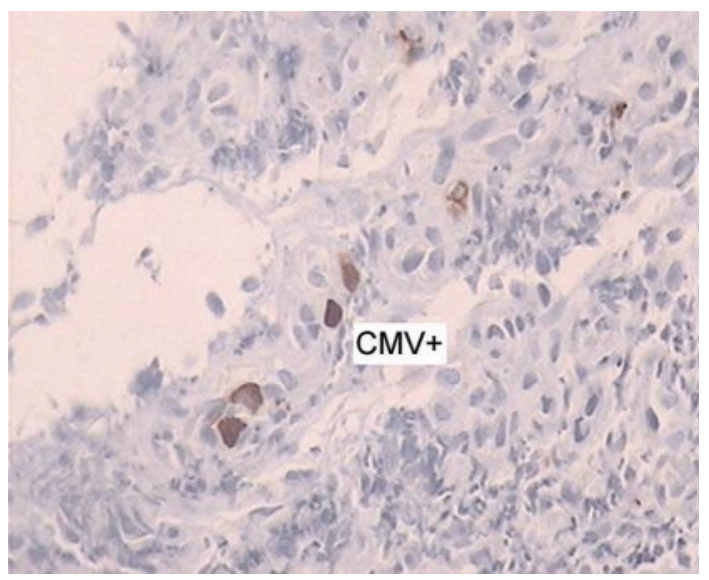




\section{References}

1 McGowan CE, Carlsten J, Bhattacharya B: Cytomegalovirus gastritis. Gastrointest Endosc 2008;68:370-371; discussion 371.

2 Moustafellos P, Hadjianastasiou V, Gray D: Postural epigastric pain as a sign of CMV gastritis: a case report. Transplant Proc 2006;38:1357-1358.

-3 Minami H, Matsushita T, Sugihara T, Kodera Y, Sakai S, Shimokata K: Cytomegalovirus-induced gastritis in a bone marrow transplant patient. Jpn J Med 1990;29:433-435.

-4 Fujishima N, Hirokawa M, Fujishima M, Saitoh H, Odashima M, Nanjo H, Sawada K: Cytomegalovirus-associated granulomatous gastritis after cord blood transplantation for acute myeloid leukemia. Int J Hematol 2007;85:362-363.

-5 Unluturk U, Aksoy S, Yonem O, Bayraktar Y, Tekuzman G: Cytomegalovirus gastritis after rituximab treatment in a non-Hodgkin's lymphoma patient. World J Gastroenterol 2006;12:1978-1979.

-6 Vergara M, Herrero J, de Torres I, Armengol JR, Saperas E, Malagelada JR: Gastric ulcers as the only manifestation of infection by cytomegalovirus in immunocompetent patients. Gastroenterol Hepatol 1998;21:332-334.

7 Setakhr V, Muller G, Hoang P, Lambert AS, Geubel A:

Cytomegalovirus-associated protein losing gastropathy in an immunocompetent adult: a case report. Acta Gastroenterol Belg 2007;70:296-299.

-8 Mohammed AA, Benmousa A, Almeghaiseeb I, Alkarawi M: Gastric outlet obstruction. Hepatogastroenterology 2007;54:2415-2420.

-9 Hinnant KL, Rotterdam HZ, Bell ET, Tapper ML: Cytomegalovirus infection of the alimentary tract: a clinicopathological correlation. Am J Gastroenterol 1986;81:944-950.

10 Lazzarotti T, Dal Monte P, Boccuni MC, Ripalti A, Landini MP: Lack of correlation between virus detection and serologic tests for diagnosis of active cytomegalovirus infection in patients with AIDS. J Clin Microbiol 1992;30:10271029.

11 Péter A, Telkes G, Varga M, Sárváry E, Kovalszky I: Endoscopic diagnosis of cytomegalovirus infection of upper gastrointestinal tract in solid organ transplant recipients: Hungarian single-center experience. Clin Transplant 2004;18:580-584.

-12 Farman J, Lerner ME, Ng C, Balthazar E, Megibow A, Herlinger H, Grimes M: Cytomegalovirus gastritis: protean radiologic features. Gastrointest Radiol 1992;17:202-206.

13 Van Thiel DH, Gavaler JS, Schade RR, Chien MC, Starzl TE: Cytomegalovirus infection and gastric emptying. Transplantation 1992;54:70-73.

14 Nowak TV, Goddard M, Batteiger B, Cummings OW: Evolution of acute cytomegalovirus gastritis to chronic gastrointestinal dysmotility in a nonimmunocompromised adult. Gastroenterology 1999;116:953-958.

15 Macdonald PS, Keogh AM, Marshman D, Richens D, Harvison A, Kaan AM, Spratt PM: A double-blind placebo-controlled trial of low-dose ganciclovir to prevent cytomegalovirus disease after heart transplantation. J Heart Lung Transplant 1995;14:32-38. 